Meynell, G. G. (1958). J. gen. Microbiol. 19, 380-389

\title{
The Effect of Sudden Chilling on Escherichia coli
}

\author{
BY G. G. MEYNELL* \\ Department of Bacteriology, Postgraduate Medical School of London
}

\begin{abstract}
SUMMARY: As found by earlier workers, Escherichia coli, growing in broth at $37^{\circ}$ was rendered incapable of gross multiplication either on nutrient agar or in nutrient broth by sudden cooling in many diluents at $4^{\circ}$. Killing is due to the joint action of a suitable diluent and of sudden chilling, since survival was complete either after sudden chilling in $0.3 \mathrm{M}$-sucrose or after gradual cooling in a potentially lethal diluent, such as Ringer's solution. Organisms in the stationary phase of growth were completely resistant. The susceptibility of growing organisms to sudden chilling changed rapidly during the exponential phase. Comparison with the survival after exposure to streptomycin, another bactericidal agent which has no effect on stationary phase cultures, showed that survival after chilling was not due to a fraction of the population being in the stationary phase.

Sudden cooling of Escherichia coli, strain B, infected with phage T 2, had the same effect as ultrasonic disruption; namely, destruction of infective centres in the first half of the latent period followed, in the second half, by release of intracellular mature phage.
\end{abstract}

When a broth culture of Escherichia coli growing exponentially (i.e. logarithmically) at $37^{\circ}$ is suddenly added to a suitable diluent at $4^{\circ}$, the majority of the bacteria are subsequently unable to form colonies on nutrient agar. A stationary phase culture is unaffected by this treatment (Sherman \& Cameron, 1934). Apart from the report by Hegarty \& Weeks (1940), who described cyclical fluctuations in susceptibility during the exponential phase of growth which were attributed to partial synchronization of division, no other investigations of this phenomenon have been traced. The present paper describes means by which survival can be either increased or decreased; the conditions under which spontaneous changes in resistance occur and their relation to other examples of phenotypic resistance to antibacterial agents; and the use of sudden chilling for inducing premature lysis of phage-infected bacteria.

\section{METHODS}

Organisms. A prototrophic strain of Escherichia coli isolated from infected urine was used in all experiments save those using phage-infected bacteria, where $E$. coli, strain B, infected with phage T2, was used.

Media. Liquid media were either broth containing $0.5 \%(\mathrm{w} / \mathrm{v})$ Difco yeast extract and $2 \%(w / v)$ Difco Bacto Casamino Acids Technical in distilled water, or the glucose + salts medium described by Hershey (1955) with

* Present address: Department of Bacteriology, St Thomas's Hospital Medical School, London, S.E. 1. 
$\mathrm{KH}_{2} \mathrm{PO}_{4}$ replaced by $1.52 \%(\mathrm{w} / \mathrm{v})$ sodium glycerophosphate, the final $\mathrm{pH}$ in either medium being $7 \cdot 2-7 \cdot 4$. All liquid cultures were incubated in a waterbath at $37^{\circ}$ and were aerated by bubbling. Foaming of broth was prevented by adding a trace of Silicone Anti-Foam M 432 (I.C.I., Gloucester House, 149 Park Lane, London, W. 1) with the tip of a straight wire. This antifoam can be sterilized by autoclaving and should the emulsion break, it can be re-established by vigorous agitation. Oxoid Nutrient Broth No. 2 (Oxo Ltd., 16 Southwark Bridge Road, London, S.E. 1), solidified with $1.5 \%$ (w/v) New Zealand agar, was used for solid medium, any additions being made after the medium had been melted and cooled to $50^{\circ}$.

Counts. All colony counts were made by spreading samples of $0.2 \mathrm{ml}$. from successive tenfold dilutions on well-dried plates, one plate being inoculated from each dilution. Each point was thus derived from a count of up to 300 colonies. Viable counts of unchilled samples were made by dilution in fullstrength Ringer's solution at room temperature, while those of chilled samples were made in the diluent used for chilling. The term 'survival' refers to the fraction, colony count after chilling/colony count on nutrient agar before chilling; while the term 'efficiency of plating on medium $A$ ' is the fraction, colony count on $\mathbf{A} /$ colony count on nutrient agar. The rate of growth of normal cultures was also estimated by means of a nephelometer (Evans Electroselenium Ltd., Harlow, Essex). Conventional techniques were used in the experiments with phage T2 (Adams, 1950).

Chilling. Bottles containing 50 or $100 \mathrm{ml}$. diluent were placed either next to or in the freezing chamber of a domestic refrigerator in order to cool the diluent to $+4^{\circ}$ or $-5^{\circ}$, respectively. Viable counts on chilled samples were done 3-4 hr. after chilling which allowed ample time for survival to become approximately constant.

\section{RESULTS}

\section{Experiments with normal bacteria}

Degree of survival after chilling. The upper part of Fig. 1 shows the growth curve of a broth culture at $37^{\circ}$, increasing with minimum doubling time of 20 min., while the lower part shows the survival on nutrient agar and on deoxycholate agar $4 \mathrm{hr}$. after dilution $1 / 100$ in quarter-strength Ringer's solution at $4^{\circ}$. The inoculum came from a culture grown overnight at $37^{\circ}$ and Fig. 1 shows that these organisms, which were presumably in the stationary phase of growth, and also those chilled after $4.5 \mathrm{hr}$. incubation, were unharmed by sudden chilling. However, in the middle of the exponential phase at $2 \mathrm{hr}$. after inoculation, survival was less than $2 \times 10^{-4}$ on nutrient agar. Colony counts did not differ significantly from counts made in broth by the dilution method. On the other hand, as found by Sherman \& Cameron (1934), no killing occurred when organisms in the exponential phase were cooled slowly by allowing $30 \mathrm{~min}$. for the temperature to fall from $37^{\circ}$ to $4^{\circ}$.

The organisms which survived sudden chilling nevertheless showed signs of damage since their efficiency of plating (see Methods) on nutrient agar containing cationic detergents was considerably less than that of either unchilled 
or slowly chilled organisms (Figs. 1, 2); i.e. these detergents reduced the colony count of organisms surviving chilling far more than that of either unchilled or slowly chilled organisms. Similar values were obtained with survivors plated on nutrient agar containing either $\mathbf{0 . 2 5} \%(\mathrm{w} / \mathrm{v})$ sodium deoxycholate or $\mathbf{1} / \mathbf{4 0}$ $(\mathrm{v} / \mathrm{v})$ 'Teepol L' (sodium or potassium salts of alkyl sulphates supplied by Shell Chemicals Ltd., 105/109 Strand, Norman House, London, W.C. 2),

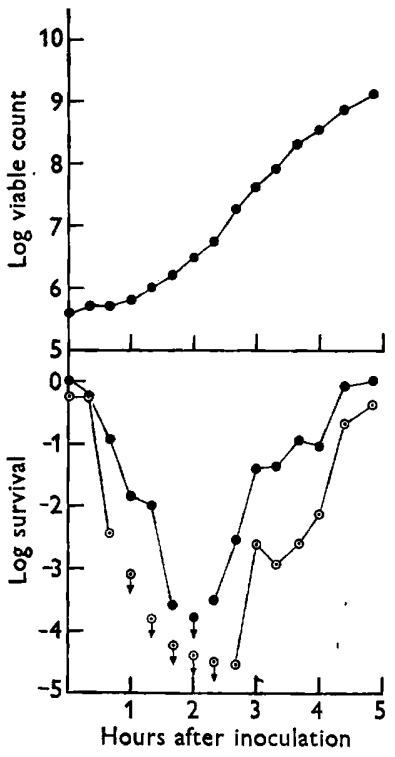

Fig. 1

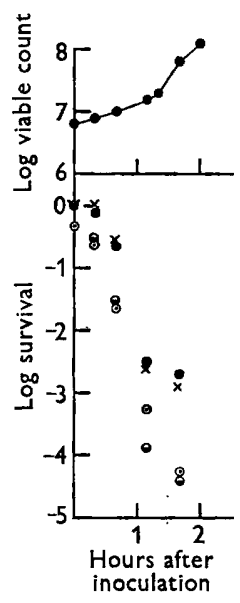

Fig. 2

Fig. 1. An overnight broth culture was diluted $1 / 2000$ in fresh broth at $37^{\circ}$. Samples of $0.5 \mathrm{ml}$. were then diluted $1 / 100$ in quarter-strength Ringer's solution at $4^{\circ}$, viable counts being made $4 \mathrm{hr}$. later on either nutrient agar (-0) or nutrient agar containing $\mathbf{0 . 2 5} \%(\mathrm{w} / \mathrm{v})$ sodium deoxycholate $(O-O)$. Points bearing arrows are derived from counts on samples in which no organisms survived to form colonies: each point gives the survival that would have been observed if one colony had been formed.

Fig. 2. The effect of various surface-active agents on survival of organisms chilled for $4 \mathrm{hr}$. in quarter-strength Ringer's solution at $4^{\circ}$. Counts were made on nutrient agar (-), and on nutrient agar containing either Tween $80(x-x)$, sodium deoxycholate $(\odot-\odot)$ or Teepol $(\ominus-\ominus)$.

minimum efficiencies of plating being $10^{-2}-10^{-3}$, whereas the minimum efficiency for unchilled cells was $0 \cdot 5-0 \cdot 1$. Neither the non-ionic detergent, Tween $80(0.5 \%$, w/v: Honeywill and Stein, 21 St James St., London, S.W. 1) nor the anionic detergents, cetyl trimethyl ammonium bromide $(\leqslant 0 \cdot 1 \%, w / v)$ or benzalkonium chloride $(\leqslant 0.02 \%, w / v)$ had any effect. The anionic detergents may have been inactivated by the medium since a heavy precipitate formed with the higher concentrations.

Organisms multiplying exponentially in glucose + salts medium at $37^{\circ}$ with doubling-time of $1 \mathrm{hr}$. were far more resistant to sudden chilling in distilled water, the minimum survival after $4 \mathrm{hr}$. being 0.5 on nutrient agar and 0.1 on the same medium containing $\mathbf{0 \cdot 2 5} \%(\mathrm{w} / \mathrm{v})$ sodium deoxycholate. The effect of 
adding peptone (final concentration of $0 \cdot 1-1 \%, w / v)$ to exponentially increasing cultures in glucose + salts medium was also measured. The growth rate did not change for $c .35 \mathrm{~min}$. after the peptone was added and during this period, the cells became completely resistant to chilling. The growth rate then increased and, concurrently, resistance to chilling declined, reaching a minimum c. $1.5 \mathrm{hr}$. later. A similar experiment was done using a mixture of prototrophic and auxotrophic organisms distinguished by their differing ability to

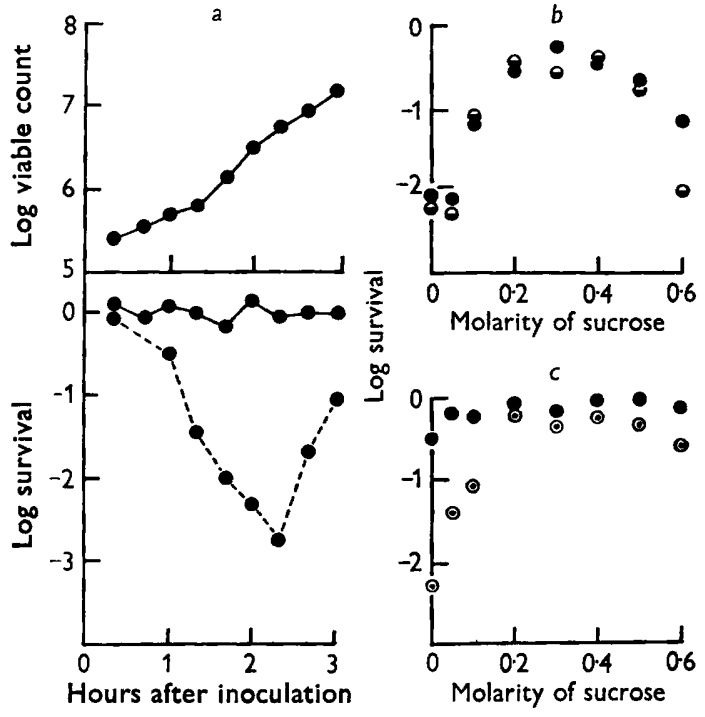

Fig. 3

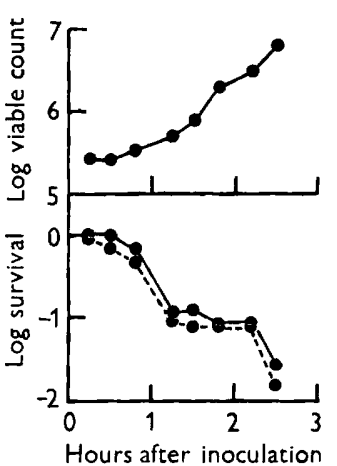

Fig. 4

Fig. 3a. Survival on nutrient agar after $4 \mathrm{hr}$. chilling in either quarter-strength Ringer's solution (O- -O) or $0.3 \mathrm{M}$-sucrose (O-O).

Fig. $3 b$ and $c$. Survival of 2 samples from growing broth cultures after $4 \mathrm{hr}$. chilling in various concentrations of sucrose at $4^{\circ}$. Counts were made on nutrient agar $(0)$ and on nutrient agar containing either $0.25 \%(w / v)$ sodium deoxycholate $(\odot)$ or $0.3 \mathrm{M}$-sucrose (ө).

Fig. 4. Survival on nutrient agar $4 \mathrm{hr}$. after sudden chilling either $(a)$ by dilution $1 / 100$ in distilled water at $4^{\circ}(-)$, or $(b)$ by dilution $1 / 2$ in broth supercooled to $-5^{\circ}$, the mixture (at a temperature of $+6^{\circ}-8^{\circ}$ immediately after mixing) being transferred to a water-bath at $+4^{\circ}(0--0)$.

ferment lactose and differing resistance to streptomycin. The susceptibility of both strains to chilling increased at virtually the same rate after the addition of peptone.

The influence of the diluent on survival after chilling. Susceptible organisms were completely unharmed by sudden chilling in sucrose, $0.3 \mathrm{M}$ being optimal (Fig. 3). Partial protection was usually given by $10-16 \%(\mathrm{w} / \mathrm{v})$ propylene glycol 4000 (range of molecular weight 3300-3600 supplied by Oxirane Ltd., 170 Piccadilly, London, W. 1) dissolved in $0 \cdot 1 \mathrm{M}$-Sørensen buffer at $\mathrm{pH} 6 \cdot 8$; by full strength Ringer's solution; and occasionally by broth. Sudden cooling of an exponential-phase culture by addition to an equal volume of broth supercooled to $-5^{\circ}$ was found to give the same survival as dilution $1 / 100$ in distilled water at $4^{\circ}$ (Fig. 4). 
The appearance of chilled cultures. No morphological differences were found between formolized samples of unchilled cultures and formolized samples of cultures in which a majority of organisms had been killed by addition to an equal volume of saline at $-5^{\circ}$. The total count determined by phase-contrast microscopy using a Helber chamber was essentially unchanged (Table 1); no differences in turbidity were seen by naked eye; no differences were detected by either phase-contrast or dark-ground microscopy of individual organisms or by electron microscopy of samples $2-4$ described in the table.

\section{Table 1. Comparison of total and viable counts on chilled cultures}

\begin{tabular}{|c|c|c|c|c|c|c|}
\hline \multirow[b]{2}{*}{ Sample } & \multicolumn{2}{|c|}{$\begin{array}{c}\text { Colony count } / \mathrm{ml} \text {. on } \\
\text { nutrient agar }\end{array}$} & \multirow[b]{2}{*}{ Survival } & \multicolumn{2}{|c|}{ Total count } & \multirow{2}{*}{$\begin{array}{c}\text { Ratio: } \\
\text { Total counts } \\
\text { after/before } \\
\text { chilling }\end{array}$} \\
\hline & $\begin{array}{l}\text { Before } \\
\text { chilling }\end{array}$ & $\begin{array}{l}\text { After } \\
\text { chilling }\end{array}$ & & $\begin{array}{l}\text { Before } \\
\text { chilling }\end{array}$ & $\begin{array}{l}\text { After } \\
\text { chilling }\end{array}$ & \\
\hline $\mathbf{1}$ & $8.1 \times 10^{7}$ & $4.56 \times 10^{6}$ & $0 \cdot 15$ & $2.42 \times 10^{7}$ & $2 \cdot 17 \times 10^{7}$ & 0.90 \\
\hline 2 & $3.7 \times 10^{7}$ & $4.5 \times 10^{6}$ & $0 \cdot 12$ & $5.76 \times 10^{7}$ & $5 \cdot 13 \times 10^{7}$ & $0 \cdot 89$ \\
\hline $\mathbf{3}$ & $5 \times 10^{7}$ & $6.5 \times 10^{6}$ & $0 \cdot 13$ & $4.88 \times 10^{7}$ & $4.44 \times 10^{7}$ & 0.91 \\
\hline 4 & $8 \times 10^{7}$ & $2.75 \times 10^{7}$ & $0 \cdot 34$ & $8.05 \times 10^{7}$ & $8.67 \times 10^{7}$ & 1.08 \\
\hline
\end{tabular}

Samples were chilled by addition to an equal volume of saline supercooled to $-5^{\circ}$, the mixtures being at once transferred to a water bath at $4^{\circ}$ for $4 \mathrm{hr}$. After a viable count had been made, formalin was added to $0.5 \%$, a portion taken for a total count, and the remaining suspension centrifuged, the deposit being resuspended in $1 / 10 \mathrm{vol}$. $0.5 \%$ formalin for examination by electron microscopy. Unchilled samples were mixed with an equal volume of $1 \%$ formalin at $20^{\circ}$ and $4 \mathrm{hr}$. later, after a total count had been made, were concentrated in the same way.

The significance of phenotypic resistance to sudden chilling. When stationaryphase organisms were added to fresh broth, susceptibility to chilling usually increased after $20 \mathrm{~min}$. (Fig. 1). However, maximum susceptibility did not develop until 1.6 hr. later. Similarly, susceptibility usually began to fall when the viable count reached $5 \times 10^{7} / \mathrm{ml}$, , that is, about $1 \mathrm{hr}$. before any fall in the rate of increase of the culture was detectable by either colony counts or nephelometry. If the initial count was too great (say, more than $5 \times 10^{7} / \mathrm{ml}$.) there was too little time for maximum susceptibility to develop as the early phase of increasing susceptibility merged with the later phase when susceptibility fell.

Susceptibility presumably develops gradually because the organisms of the stationary phase inoculum differ in the time taken to become sensitive to chilling. In the second half of the growth curve, susceptibility presumably falls because, as the concentration of organisms increases, the mean rate of growth of the culture falls, leading to a change in survival. The survivors might have comprised a non-multiplying fraction of the culture, but this possibility was excluded by comparing the survival after chilling with that following exposure to a relatively high concentration of streptomycin, another agent which kills rapidly dividing organisms but which has no effect on organisms in the stationary phase of growth (Garrod, 1948; Mitchison \& Selkon, 1956). If a fraction of the bacterial population was non-dividing, the ultimate survival after either chilling or exposure to streptomycin should be 
the same. Figure 5 shows the effect of streptomycin on samples taken at various times in the second half of the growth curve. Curves $A$ and $B$ do not differ essentially; $C$ shows a resistant fraction which will be considered in the Discussion; and $D$ has the same form as $A$ and $B$. The next six curves show a progressive increase in the resistance of the culture until the population is finally completely resistant. Comparison of Figs. 1 and 5 shows that when
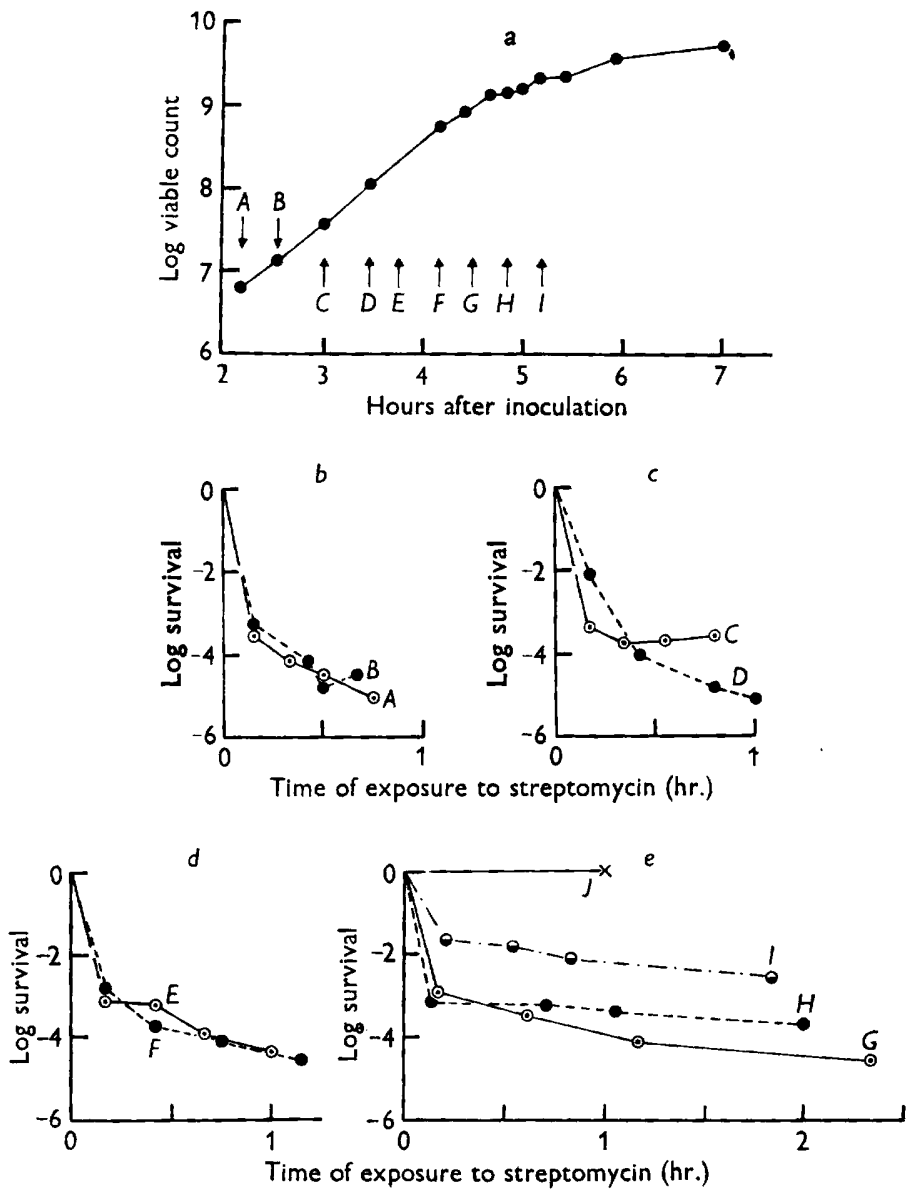

Fig. 5. Effect of streptomycin on a culture in the second half of the growth curve. At times $A, B \ldots$, shown in Fig. $5 a, 4 \mathrm{ml}$. of culture growing with aeration at $37^{\circ}$ were transferred to $0.44 \mathrm{ml}$. streptomycin solution at $37^{\circ}$, aeration being continued. The final concentration of streptomycin was $200 \mu \mathrm{g} . / \mathrm{ml}$. Counts were made at intervals on nutrient agar yielding the curves $A, B, \ldots$, shown in Figs. $5 b-e$. Curve $J$ was determined on an overnight culture.

the colony count was $10^{8 \cdot 5}$, survival after chilling was $0 \cdot 1$, while after exposure to streptomycin for $1 \mathrm{hr}$. it was $\mathbf{0 \cdot 0 0 0 1}$. In another experiment, samples of the same culture were either chilled or treated with streptomycin, and again resistance to chilling was virtually complete at times when the culture was still highly susceptible to streptomycin (Fig. 6). 


\section{Experiments with Escherichia coli infected with phage T2}

Doermann (1952) and Anderson \& Doermann (1952) showed by disruption of phage-infected bacteria that intracellular mature phage first appeared about half-way through the latent period. These authors used either 'lysis from without', produced by the addition of a high multiplicity of phage T6 acting in the presence of $0.01 \mathrm{M}$-potassium cyanide (Doermann, 1952), or ultrasonic

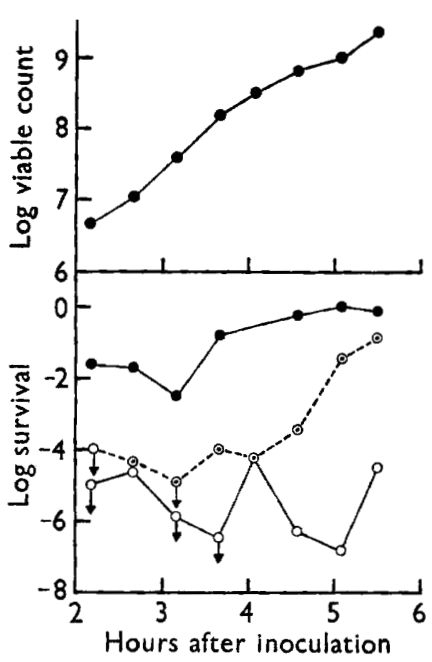

Fig. 6

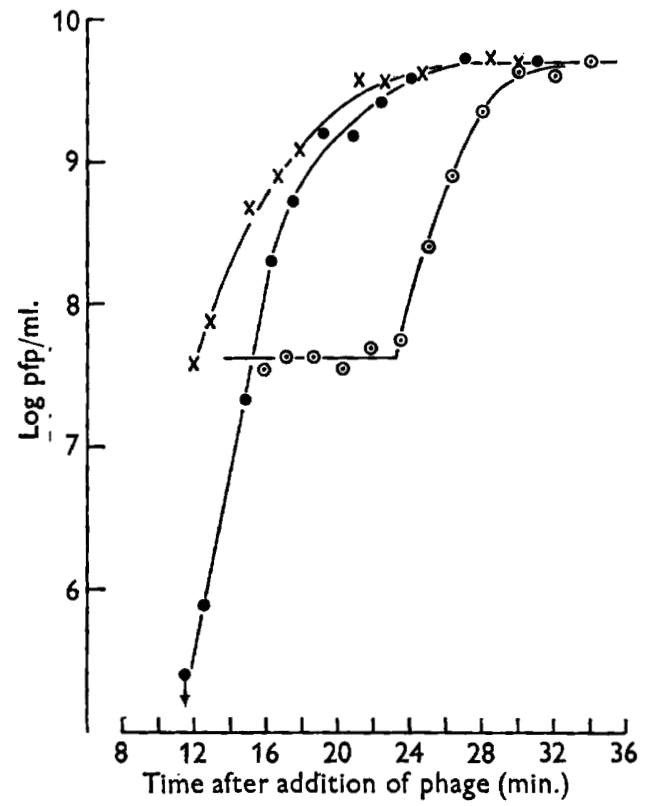

Fig. 7

Fig. 6. Survival (a) $4 \mathrm{hr}$. after sudden chilling by dilution $1 / 100$ in distilled water at $4^{\circ}$, counts being made on nutrient agar with $\left(\odot-\odot^{-}\right)$and without $(-0) 0.25 \%$ sodium deoxycholate; and $(b) 1 \mathrm{hr}$. after mixture with streptomycin solution at $37^{\circ}$ at a final concentration of $200 \mu \mathrm{g} . / \mathrm{ml}$. $(\mathrm{O}-\mathrm{O})$.

Fig. 7. Effect of sudden chilling on Escherichia coli, strain B, infected with phage T2. An overnight culture was diluted $1 / 2000$ in fresh broth at $37^{\circ}$, phage T2 being added after $3.5 \mathrm{hr}$. incubation. Antiphage serum was added $5 \mathrm{~min}$. later. At $10 \mathrm{~min}$., when $>99 \%$ unabsorbed phage was neutralized, the culture was diluted sufficiently to prevent inactivation of liberated phage by either serum or reabsorption. The multiplicity of infection was 1.5. Samples were chilled by dilution $1 / 100$ in distilled water at $4^{\circ}$ and were counted $4 \mathrm{hr}$. later $(-)$ ). Other samples were diluted $1 / 10$ in $0.01 \mathrm{M}$-potassium cyanide in broth at $37^{\circ}$ and were counted after 40-60 min. $(x-x)$. The curve $(\odot-\odot)$ is a control one-step growth curve on the same culture.

disintegration (Anderson \& Doermann, 1952). After mature intracellular phage has appeared, 0.01 M-potassium cyanide alone will promptly induce lysis and gives results identical with those of the first method (Doermann, 1952).

Figure 7 shows the amount of phage released during the latent period either by $0.01 \mathrm{M}$-cyanide or by sudden chilling in distilled water at $4^{\circ}$. Chilling 
is evidently as efficient as cyanide in extracting phage from the infected bacteria, for the counts by either method are the same at the end of the latent period (24 min. onwards) and are in turn the same as the counts obtained after spontaneous lysis. Before 24 min., the curve for chilled samples resembles that obtained by ultrasonic disintegration since at any given time, the counts are always lower than those obtained by treatment with cyanide (presumably because cyanide does not stop phage maturation instantaneously) and since less than $1 \%$ of infected bacteria survive chilling, whereas $c .50 \%$ are known to survive cyanide. Other experiments showed that sudden chilling led to maximum phage release within the succeeding 2 min. so that chilling must have acted by causing almost immediate lysis and not by arresting phage growth, followed by lysis at the normal time. Chilling in $0.3 \mathrm{M}$-sucrose at $4^{\circ}$ gave the same counts as chilling in distilled water.

\section{DISCUSSION}

These experiments show that killing of an exponential phase culture is not due to sudden cooling in itself (since survival was complete in $0.3 \mathrm{M}$-sucrose), but evidently requires a suitable diluent as well as a sudden fall in temperature. The second condition suggests that survival after slow cooling in a potentially lethal diluent follows adaptation to the new medium by a mechanism having a negligible action at $4^{\circ}$. The first condition suggests that this mechanism prevents entry of water into the cell since survival was complete in sucrose, known to have this action since it can prevent plasmoptysis and lysis of protoplasts of Escherichia coli (Lederberg, 1956). However, bacterial death following chilling was not accompanied by lysis, for chilled and unchilled samples from the same culture did not show any visible differences. The effect of sudden chilling on phage-infected bacteria is presumed to differ from its effect on normal bacteria since it was not modified by chilling in $0.3 \mathrm{M}$-sucrose. Maaløe (1950) reported that when Escherichia coli, strain B/1, was infected with phage $\mathbf{T} 4 \mathrm{r}$ at $36^{\circ}$ in broth and subsequently was suddenly cooled by dilution in broth at $19^{\circ}$ during the last third of the latent period, some infected bacteria lysed. However, his results differ from those given above in that phage-infected bacteria were not destroyed by this degree of cooling in the first two-thirds of the latent period.

After sudden chilling of a broth culture in Ringer's solution, the survival of stationary phase organisms on nutrient agar containing $\mathbf{0 . 2 5} \%$ sodium deoxycholate was $c .0 .5$, while that of exponential phase organisms was often $10^{-5}-10^{-6}$. Unfortunately, this observation could not be applied to the isolation of nutritionally exacting organisms from a predominantly prototrophic population growing in glucose + salts medium because the latter was only slightly susceptible.

It will be seen from Fig. 1 that a culture is uniformly susceptible to chilling for only a small part of the exponential phase of growth. At other times, susceptibility is either increasing or decreasing. Such heterogeneity in resistance could be accounted for by postulating pre-existent heterogeneity in 
the population as would occur, for example, if the organisms differed in their requirement for some constituent of the medium so that as the count increased and the concentration of this nutrient fell, the growth rate of a fraction of the organisms fell to such an extent that it became resistant to chilling. The simplest form of this hypothesis, which supposes the survivors to comprise a non-multiplying fraction of the population, is excluded by the differing survival after sudden chilling and after exposure to streptomycin (Figs. 1, 5 and 6). It might be argued that at the moment of exposure to streptomycin, the size of the phenotypically streptomycin-resistant fraction was the same as that resistant to chilling but that it rapidly became phenotypically sensitive following death of the initially sensitive fraction. This seems improbable if depletion of a growth factor led to phenotypic resistance but is more plausible if this arose from oxygen lack. However, this is also unlikely for Paine \& Clark (1953) have shown that after addition of streptomycin to susceptible cultures, oxygen consumption continues virtually unchanged for at least $1 \mathrm{hr}$. although more than $95 \%$ cells may be incapable of colony formation.

The killing curves after either chilling or streptomycin treatment usually appeared to be smoothly concave so that the distribution of resistance to both agents could be continuous in the population. A completely resistant fraction was observed in one experiment (Fig. $5 c$, curve $C$ ) and it is supposed that this subculture was unfavourable to growth so that part of the population rapidly became phenotypically resistant. Differences between subcultures are presumably responsible for the gross differences in survival shown by the various subcultures treated with streptomycin in the second experiment (Fig. 6).

Several other bactericidal agents are known to act only on actively dividing cultures and, as seen above, a considerable fraction of the bacterial population may survive by virtue of its phenotype although it is clearly potentially (i.e. genotypically) sensitive. The survivors will then appear fully sensitive to the agent when retested by any method entailing multiplication (e.g. the usual antibiotic sensitivity tests). A well-known example of this phenomenon is provided by the action of penicillin on Staphylococcus aureus (Bigger, 1944); while Mitchison \& Selkon (personal communication) pointed out that similar results are obtained with this species and streptomycin. Garrod (1948, fig. 6), also using streptomycin and $S$. aureus, reported a curve resembling curve $C$ in Fig. 5 and found the members of the surviving fraction to be no more resistant than the original population in subsequent sensitivity tests. Survival curves showing resistant fractions of varying sizes are also obtained after exposure of cultures of Escherichia coli to ultraviolet light (Dr T. Alper, personal communication). In this system, as in the experiments with chilling, the behaviour of the culture can be forecast fairly well from the initial bacterial count and the time of incubation. On the other hand, little help is given by the observed rate of multiplication for (to take the extreme case where the resistant fraction was non-multiplying) the resistant fraction of the total population could clearly change from $10^{-2}$ to $10^{-6}$, for example, without producing a detectable change in the rate of growth of the culture as a whole. 
I should like to thank Dr R. C. Valentine for examining samples of chilled cells by electron microscopy, and I.C.I. Ltd. and Oxirane Ltd. for gifts of antifoam and of propylene glycol, respectively.

\section{REFERENCES}

Adams, M. H. (1950). Methods of study of bacterial viruses. Meth, med. Res. $2,1$. Anderson, T. F. \& Doermann, A. H. (1952). The intracellular growth of bacteriophages. II. The growth of T 3 studied by sonic disintegration and by T6cyanide lysis of infected cells. J. gen. Physiol. 35, 657.

BigGer, J. W. (1944). Treatment of staphylococcal infections with penicillin by intermittent sterilization. Lancet, ii, 497.

DoermanN, A. H. (1952). The intracellular growth of bacteriophages. I. Liberation of intracellular bacteriophage T4 by premature lysis with another phage or with cyanide. J. gen. Physiol. 35, 645.

Garrod, L. P. (1948). The bactericidal action of streptomycin. Brit. med. J. i, 382.

Hegarty, C. P. \& Weers, O. B. (1940). Sensitivity of Escherichia coli to cold-shock during the logarithmic growth phase. J. Bact. 39, 475.

Hershey, A. D. (1955). An upper limit to the protein content of the germinal content of bacteriophage T2. Virology, 1, 108.

Lederberg, J. (1956). Bacterial protoplasts induced by penicillin. Proc. nat. Acad. Sci., Wash. 42, 574.

MAALøE, O. (1950). Some effects of changes in temperature on intracellular growth of the bacterial virus T4r. Acta path. microbiol. scand. 27, 680.

Mrtchison, D. A. \& Seikon, J. B. (1956). The bactericidal activities of antituberculous drugs. Amer. Rev. Tuberc. 74, 109.

Paine, T. F. \& Clark, L. S. (1953). The effect of streptomycin on oxygen uptake and viability of resting suspensions of Escherichia coli. Science, 118, 73.

Sherman, J. M. \& Cameron, G. M. (1934). Lethal environmental factors within the natural range of growth. J. Bact. 27, 341.

(Received 15 April 1958) 